\title{
Research Square \\ Thermal and Mechanical Stabilities of Core-shell Microparticles Containing a Liquid Core
}

\section{Fariba Malekpour Galogahi}

Griffith University

\section{Hongjie An}

Griffith University

\section{Yong Zhu}

Griffith University https://orcid.org/0000-0001-6701-5694

Nam-Trung Nguyen ( $\nabla$ nam-trung.nguyen@griffith.edu.au )

Griffith University https://orcid.org/0000-0003-3626-5361

\section{Research Article}

Keywords: core-shell particle, liquid core, thermal stability, mechanical stability, microfluidics

Posted Date: September 15th, 2021

DOI: https://doi.org/10.21203/rs.3.rs-870641/v2

License: (c) (i) This work is licensed under a Creative Commons Attribution 4.0 International License.

Read Full License

Version of Record: A version of this preprint was published at Journal of Molecular Liquids on December 1st, 2021. See the published version at https://doi.org/10.1016/j.molliq.2021.117726. 


\title{
Thermal and Mechanical Stabilities of Core-shell Microparticles Containing a Liquid Core
}

Fariba Malekpour Galogahi, Hongjie An, Yong Zhu, Nam-Trung Nguyen*

\author{
Queensland Micro- and Nanotechnology Centre, Griffith University, 170 Kessels Road, \\ Nathan, 4111 Queensland, Australia \\ E-mail: nam-trung.nguyen@griffith.edu.au
}

\section{Abstract}

Thorough understanding of the behaviour of core-shell microparticles with a liquid core is essential for determining their performance in applications under different operation conditions. This paper reports the behaviour of core-shell particles with a liquid core under thermal and mechanical loads. First, we formulated an analytical model for the heating process of a coreshell microparticle with a liquid core. Next, we utilised an axisymmetric model of an elastic spherical shell upon compression to describe the deformation of a core-shell microparticle. Finally, we conducted experiments to validate these models. Both thermal and mechanical models agree well with the experimental data. The maximum temperature a core-shell microparticle can withstand depends on the liquid, the geometry, and the material of the shell. The critical compression force before rupture of a core-shell microparticle depends on the Poisson's ratio of the shell material and the shell thickness relative to the outer shell radius. The rupture force and rupture temperature increase with increasing shell thickness.

Keywords: core-shell particle, liquid core, thermal stability, mechanical stability, microfluidics

\section{Introduction}

Recently, core-shell microparticles have attracted increasing attention from the research community due to the relatively simple manipulation, versatile choice of materials, and modifiable surface properties, as well as their broad range of applications in biomedicine, biotechnology, and chemistry [1]. For instance, encapsulation is a promising technique for modern laundry powders, which allow the co-existence of enzyme and surfactants, enabling 
fragrance molecules to stay with clothes during and after washing, and keep bleaches stable and effective. Microcapsules packed with a small amount of liquid core are used as micro-reactors, storage containers, and delivery carrier for food, drug, coating, and cosmetic products [2]. These microcapsules are usually formed by single emulsion or double emulsion techniques such as magnetic stirring [3], vortex mixing [4] electro-spraying [5], and microfluidics. Digital microfluidics is remarkably efficient compared to other techniques in terms of producing stable and monodispersed core-shell microcapsules. The formation process of double emulsion can be controlled on chip with integrated control system [6], temperature sensing and control [7, 8], and enhanced heat ad mass transfer [9]. Furthermore, many applications require the controlled release of the liquid core from the microcapsules [2]. Some microcapsules for drug delivery or implant are designed to be permeable and liquid core can be released continuously [10]. However, a porous shell could be a problem for storage, limiting its use for encapsulating small molecules [11]. Other core-shell microcapsules are designed to be impermeable and only release their contents by rupture, such as mechanical friction or compression [12], $\mathrm{pH}$ change [13], enzymatic degradation, and thermal stress $[14,15]$.

Understanding the mechanical and thermal properties of core-shell microparticles is crucial for their design, synthesis, handling, and application. First, thermal properties of both core and shell materials determine the operation temperature for applications such as storage, cargo delivery, sensing, and cell culture [16]. Thermal curing of the shell can be problematic for a volatile liquid core or solvent with low boiling temperature [14]. Packing an organic core in an inorganic shell can improve its thermal stability [17]. Generally, preious studies suggest three possible mechanisms that control the thermally induced release of core contents from microcapsules: (i) the burst of the shell due to the high thermal expansion coefficient of liquid core and subsequently increased internal thermal stresses [18, 19]; (ii) the permeation and diffusion of the core contents through the porous or permeable microcapsule shell; and (iii) the 
degradation of microcapsule shell at the high temperature [19]. The release mechanism is an essential factor for later applications, for which, e.g., the thermal stability of shell as a thermal protection layer is important or controlled release of core components at a specific temperature is desirable. The thermal stability is correlated with thermal tolerance of microcapsules in the actual applications such as the temperature of storage and delivery of cargo, triggering temperature in catalysis, and thermal tolerance of sensing systems [20]. Furthermore, size and thickness of the shell affect the thermal behaviour of core-shell microparticles. The rupture temperature has been found increase exponentially with decreasing size of the microcapsules. A critical threshold size of approximately $10 \mu \mathrm{m}$ was reported by Zhao et al.[20]. However, the thermal behaviour of liquid core-shell microparticles is not yet fully understood due to the lack of a theoretical model.

Intrinsic mechanical properties of microcapsules, particularly of the shell, affect their performance. Under some circumstances, the release of encapsulated substances require stress, e.g. massage for the release of perfume for personal skin care, and compression for encapsulated ink in inkless paper and dyes in textile. The hydrodynamic shear stress in blood vessels is a major problem for microcapsules in drug delivery [21]. Core-shell capsules for scaffolds in tissue engineering, for instance in bone and cartilage, are expected to withstand a high mechanical stress [22]. A number of characterisation methods of mechanical properties of coreshell microparticles can be found in a recent review paper [23]. Compression of microcapsule of $10-\mu \mathrm{m}$ diameter were investigated by nanoindentation with an atomic force microscopy (AFM) with relatively high spatial resolution [24]. Capsules with diameters on the order of $100 \mu \mathrm{m}$ are too big to be measured by AFM, and are usually characterised by compression between two parallel plates using a micromanipulator [24]. Among these characterisation methods, compression between two flat parallel plates is an effective mean to assess the rupture of the shell, as well as the elasticity of the microcapsule. Early compression studies indicated 
that the deformation under compression largely depends on the shell material, and the rupture force increases with increasing size of the microparticles [25]. Compression of different liquid core-shell microcapsules were studied and tested [26-29] using the axisymmetric model of a thin-shell microcapsules compressed between two parallel flat plates. Bando et al. proposed the assumption of no initial stretch of the shell which caused numerical instability and developed a new model [30]. However, the model may lead to errors for thick-wall capsules, because the effect of bending stress cannot be neglected [31,32].

We previously prepared core-shell microparticles consisting of a HFE7500 fluorinated oil core and a polymer shell of trimethylolpropane trimethacrylate (TMPTMA) using dropletbased microfluidics $[33,34]$. The core-shell microparticles can be tuned for different ratios of shell thickness to outer radius. However, little information is available on thermal and mechanical properties of these particles. The present study aims to experimentally investigate the behaviour of core-shell microparticles with a liquid core during heating and compression processes. The experimental results are compared with analytical models considering parameters such as particle size and shell thickness. The thermal model predicts the stress induced by the thermal expansion of the liquid core during the heating process acting on the shell layer. The axisymmetric model of an elastic spherical shell can describe the behaviour of the core-shell microparticle under compression.

\section{Analytical models}

\subsection{Mechanical behaviour of a core-shell particle with liquid core}

Next, we considered a thick-walled spherical shell of thickness $h=b-a$ and the elastic modulus $E$ fully filled with a liquid, Fig. 1(a). For simplicity, the liquid was considered as incompressible, so the pressure was solely applied to the shell. The shell material was assumed to be homogeneous, impermeable, elastic, and isotropic. The thickness $h$ was 
assumed to remain constant without any bend or inflation during the compression process, and the internal volume of the shell remains constant as it deforms. The shell is compressed between two rigid horizontal plates, Fig. 1(b). Because of the compression, the shell deforms symmetrically with a distance $x$ from top and bottom each and a total displacement of $d=2 x$.

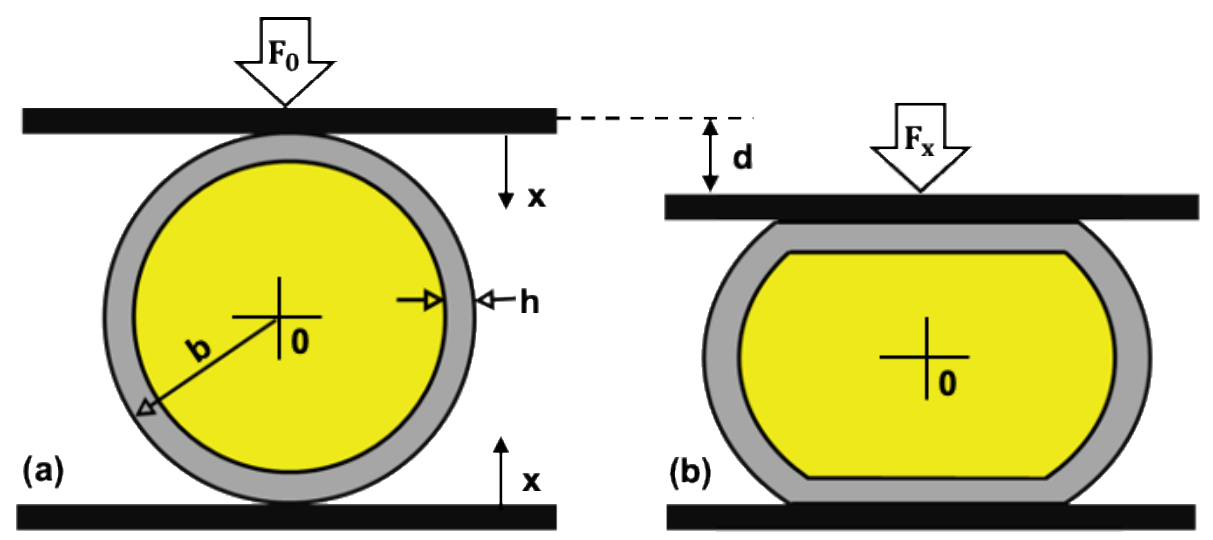

Figure 1. Compression process of a thick-walled spherical core-shell particle: (a) Initial state; (b) Deformed state.

We first used the Pauchard's model to find the elastic energy stored in the spherical elastic shell. Pauchard and Rica [35] considered two different compression regimes: (i) the first flattening cap of a spherical shell into a flat plane under low force and then (ii) axisymmetric inwards buckling of the flattened portion of shell resulted from a higher load. In the first regime, the spherical shell is flattened against the horizontal surfaces. The elastic energy of this regime is:

$U_{1}=A \frac{1}{4} \frac{E h^{5 / 2}}{b} x^{3 / 2}+B \frac{E h}{b} x^{3}$,

where $A$ and $B$ are dimensionless constants depending on the properties of the shell material through the Poisson's ratio $v$. We determined these constants experimentally through curve fitting across all experiments using core-shell particles of the same shell material but different 
geometries. The first term represents the energy that results from axisymmetric folding of the spherical shell and balancing the bending and stretching energy of the flattened surface. The second term accounts for the energy of compression and flattening out a part of the spherical shell $[35,36]$. The applied force $F$ causing the displacement $x$ from each side of the sphere can then be obtained by differentiating the elastic energy (9):

$F_{x}=A \frac{3}{8} \frac{E h^{5 / 2}}{b} x^{1 / 2}+3 B \frac{E h}{b} x^{2}$

The force $F_{x}$ is expected to depend slightly on the Poisson's ratio $v$.

The elastic energy of buckling in the second regime is:

$U_{2}=A \frac{E h^{5 / 2}}{b} x^{3 / 2}+C \frac{E h^{3}}{b} x$

The total displacement reaches a critical value $d_{\max }=2 x_{\max }$, where the shell ruptures if compression continues. The critical displacement can be determined by the continuity condition $U_{1}=U_{2}$. In the following section, we conducted experiments on liquid core-shell particles generated by our microfluidic approach to determine the constants $A, B$ and $C$ by fitting the measured force versus displacement data.

\subsection{Thermal behaviour of a core-shell particle with liquid core}

In this section, the thermal characteristics of oil core-polymer shell particles are formulated analytically. The core-shell particle is considered as a thick-walled particle because the wall thickness is greater than a tenth of overall radius of the particle. Figure 2 shows the schematic heating process of a core-shell particle and the forces acting on the spherical shell during the heating process. Gradually increasing the temperature increases the pressure of the liquid and induces a uniform pressure on the inner wall of the shell. Eventually, the pressure of the liquid 
exceeds the maximum tensile strength of the shell and causes it to rupture, Figure 2(a). For simplification and ease of presentation, the following assumptions were made for the model:

(i) The shell thickness of a core-shell particle remains constant and no curvature or slope occurs during the heating process; (ii) The core is located at the centre of the particle; (iii) Both core and shell are spherical an maintain this shape during the heating process; (iv) The volume of the rigid shell remains unchanged during the heating process; (v) Temperature distribution is uniform throughout the shell layer and the core; (vi) Pressure is uniformly distributed over the inner wall of the shell and change gradually with temperature; (v) The liquid encapsulated inside the shell is considered homogeneous and in a thermodynamic equilibrium states; and (vi) The liquid core is assumed to be in a single-phase before the rupture of the shell.

(a)

155

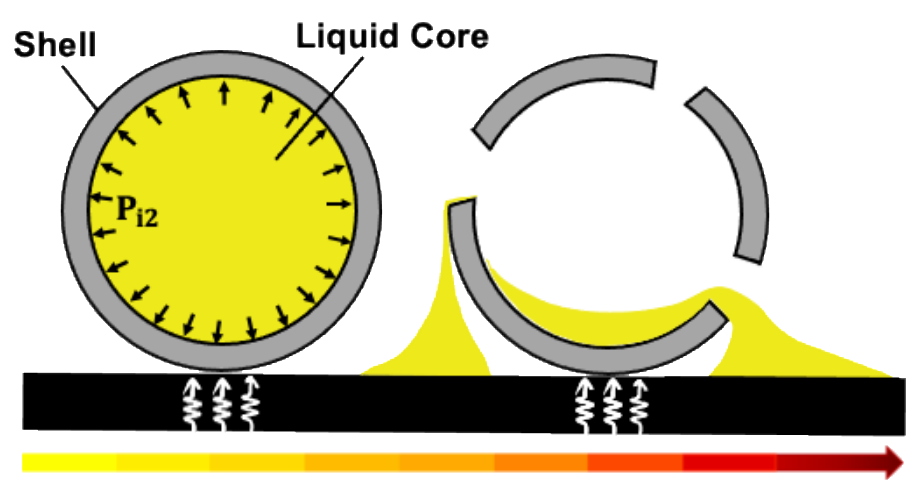

(b)

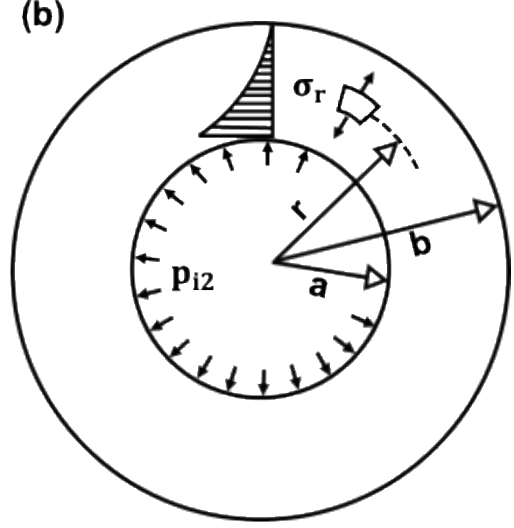

Figure 2. (a) Schematic of the process through which a liquid core-shell particle undergoes heat-induced change; (b) Model of a thick-walled spherical core-shell particle representing internal pressure and induced stress [37].

For a single-phase oil core encapsulated in a solid shell, the volume $V$, the absolute temperature $T$, and pressure $P$ are interdependent quantities. The relationships between $V, T$, and $P$ can be generally described by an equation of state [38]:

$f(P, V, T)=0$ 
$175\left(\frac{\partial P}{\partial T}\right)_{\mathrm{V}}=\frac{\alpha}{K}$ pressure:

This state equation allows for the expression of one quantity of volume, temperature, and pressure in terms of the other two. For volume $V(T, P)$ as a function of temperature and

$\mathrm{d} V=\left(\frac{\partial V}{\partial T}\right)_{\mathrm{P}} \mathrm{d} T+\left(\frac{\partial V}{\partial P}\right)_{\mathrm{T}} \mathrm{d} P$

considering a constant liquid volume $\mathrm{d} V=0$ :

$0=\left(\frac{\partial V}{\partial T}\right)_{\mathrm{P}} \mathrm{d} T+\left(\frac{\partial V}{\partial P}\right)_{\mathrm{T}} \mathrm{d} P$

and rearranging this equation for $\partial P / \partial T$ results in:

$\left(\frac{\partial P}{\partial T}\right)_{\mathrm{V}}=-\left(\frac{\partial V}{\partial T}\right)_{\mathrm{P}} /\left(\frac{\partial V}{\partial P}\right)_{\mathrm{T}}$

In (4), the partial derivatives are related to the two thermodynamic coefficients, volume expansion coefficient $\alpha$, and isothermal compressibility $K$ with $\alpha=\frac{1}{V}\left(\frac{\partial V}{\partial T}\right)_{\mathrm{P}}$ and $K=-\frac{1}{V}\left(\frac{\partial V}{\partial P}\right)_{\mathrm{T}}$, respectively. Substituting these coefficients into equation (7) results in:

Integrating both sides of (5) results in the final internal pressure $P_{i 2}$ :

$177 \quad P_{i 2}=P_{i 1}+\frac{\alpha}{K}\left(T_{2}-T_{1}\right)$,

where $P_{i 1}$ and $T_{1}$ are the initial internal pressure and temperature, which are also the ambient pressure and temperature, $P_{i 2}$ and $T_{2}$ are the internal pressure and temperature of liquid core in the final state. Considering temperature and pressure dependence, Dan et al. [39] proposed an empirical isothermal compressibility model for HFE 7500 with an isothermal compressibility $K$ :

183 $K=0.07466 /\left[\left(1-0.07466 \ln \frac{\beta+P}{\beta+0.1}\right)(\beta+P)\right]$

184 with $\beta=308.2-1.301 T+1.414 \times 10^{-3} T^{2}$ and $P$ are pressures in MPa. 
Considering a spherical thick-walled shell shown in Figure 2(b), which is filled with the liquid and subjected to the pressure induced by heating, the spherically symmetrical solution leads to the normal stress inside the shell under the internal pressure $P_{i 2}$ [37]:

$\sigma_{\mathrm{r}}=-\frac{a^{3} P_{\mathrm{i} 2}}{r^{3}} \frac{b^{3}-r^{3}}{b^{3}-a^{3}}$

Where $a$ and $b$ are the inner and outer radii of the shell, respectively.

\section{Experiment}

\subsection{Materials}

Fluorinated oil (HFE, Novec 7500 3M) was purchased from Sigma-Aldrich Chemical Reagent Co., Ltd. Ethyl- 4(dimethylamino)benzoate, camphorquinone, and trimethylolpropane trimethacrylate were purchased from Merck. All chemicals used in this study were of analytical grade and distilled water was used in all experiments.

\subsection{Fabrication of microfluidic device}

The design of the microfluidic device used to produce core-shell droplets was previously reported [33]. We fabricated the PDMS microfluidic device using soft-lithography. The master mould with microfluidic channel patterns was fabricated in SU-8 on a silicon wafer by standard photolithography. Width and height of the microchannels are $200 \mu \mathrm{m}$ and $100 \mu \mathrm{m}$, respectively. A mixture of PDMS and curing agent in a 10:1 ratio was poured into the mould master and then was degassed and cured at $75^{\circ} \mathrm{C}$ for 1 hour. The PDMS device was cut out from the mould. A biopsy punch with the same diameter as the tubing was used for punching the inlets and the outlets. The PDMS part was subsequently bonded onto glass after treating the bonding surfaces with air plasma. Finally, by passing water through the channels a hydrophilic PDMS device was obtained.

\subsection{Preparation of core-shell particles}


The PDMS device has three cross junctions: HFE7500 oil was used as the inner phase at the first junction and to disperse HFE7500 oil and produce oil core; TMPTMA was used as the middle phase at this junction, Figure 3.

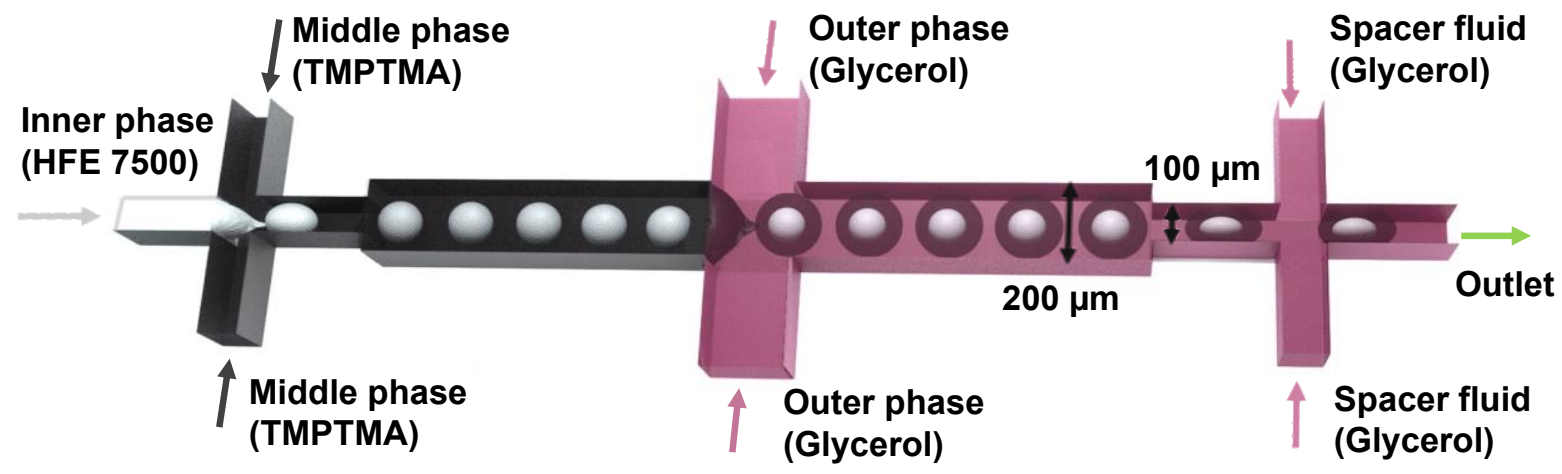

Figure 3. Schematic illustration of the flow focusing microfluidic device used for the generation of liquid core-shell microparticles.

The TMPTMA was prepared by mixing ethyl- 4(dimethylamino)benzoate $(0.06 \mathrm{~g})$, camphorquinone $(0.05 \mathrm{~g})$, and TMPTMA (10 g) using mechanical stirred at $600 \mathrm{rpm}$ for $1 \mathrm{hr}$ [40]. Distilled water with glycerol $(50 \% \mathrm{v} / \mathrm{v})$ was used as the outer liquid phase at the second junction. The core-shell droplets generated were then transferred through the channel to the third junction and were collected at the outlet. The flow rates of all fluids were controlled using syringe pumps. The flow rate of TMPTMA was kept constant at $150 \mu \mathrm{L} / \mathrm{hr}$. The flow rates of HFE7500 oil were set at 60,100 and $140 \mu \mathrm{L} / \mathrm{hr}$ at the first junction to achieve core-shell particles with shell to outer radius rations of $0.41,0.33$ and 0.21 , respectively. The flow rates of glycerol were fixed at 800 and $3,000 \mu \mathrm{L} / \mathrm{hr}$ at the second and third junctions, respectively. The formation of core-shell droplets in the PDMS device was monitored using an inverted microscope (Nikon, Eclipse Ti) connected to a computer.

At the outlet, a long tubing was used to collect the generated core-shell droplets. Since the core-shell droplets tended to aggregate while passing through the outlet tubing, the tubing was exposed to an UV light to instantaneously cross-link the shell layer. However, the particles 
did not completely polymerise due to the limited residence time. Hence, the collected particles were subsequently further exposed to UV light again for five minutes. The collected core-shell particles at the outlet were then washed once with methanol to dissolve the bulk polymer waste and to prevent particles from sticking during storage and drying. Finally, the generated coreshell microparticles were dried in a vacuum desiccator for 30 minutes. Optical microscopy images of the dried core-shell microparticles were then taken with a microscope (Eclipse Ti, Nikon).

\subsection{Mechanical behaviour of core-shell microparticles}

We characterised the mechanical properties of core-shell microparticles using compression between two parallel plates. Figure 4(a) shows a right-angle mounting adapter set up on a single-axis translation stage with a standard micrometre positioner was placed perpendicular to the pan of an analytical balance. A single core-shell particle on a microscope slide was placed on the balance pan. During the experiment, the side view of the particle was observed and recorded with a camera. The mounting adapter moved downward and compressed the coreshell microparticles. The applied load on the particles was measured using the precision balance. During the compression test, the force acting on the particle and the displacement corresponding to the deformation was recorded. Measurements were carried out for particles with different sizes. An inverted microscope (Nikon, Eclipse Ti) was used to determine the morphology and geometry of the dried core-shell microparticles were before each experiment. 

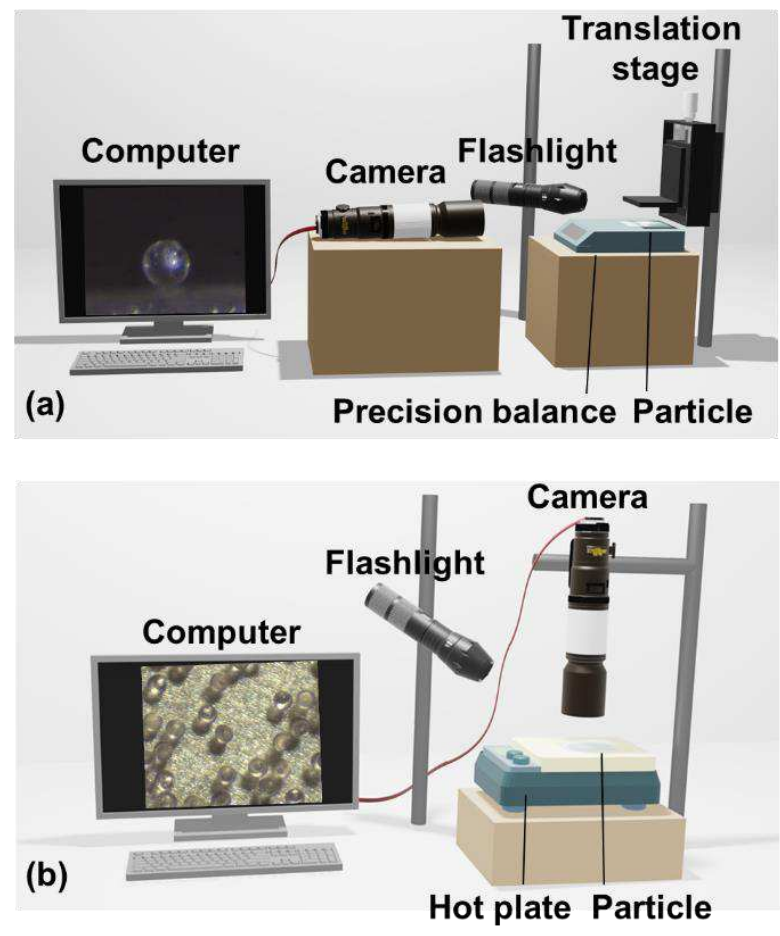

Figure 4. Schematic of the experimental setups for investigations for: (a) the thermal behaviour and (b) mechanical behaviour of core-shell microparticles with liquid core.

\subsection{Thermal behaviour of core-shell microparticles}

We carried out thermal stability experiments with a controlled hot plate, Figure 4(b). The sample was placed on a glass slide, which was then placed on the hot plate. The measurements were performed under atmospheric pressure with $5^{\circ} \mathrm{C}$ temperature increment from room temperature until a change in the core-shell particles surface was observed. For an accurate measurement, the core-shell particles were kept for 10 minutes at each temperature value. The first scan was carried out from room temperature to the critical rupture temperature, when the shell ruptures and the core liquid escapes. Three particle samples from different preparation batches were studied and the average value was recorded. The different stages of heating were recorded with a camera (Ximea xiQ-USB3 MQ013CG-ON) and a high-resolution magnification lens (Edmund Optics) placed on top of the sample.

\subsection{X-Ray Computed Microtomography (XCMT)}


High resolution X-ray computed microtomography was also used to analyse 3D microstructure of core-shell microparticles. Dried core-shell particles were placed on a cylindrical holder that was covered with a clear tape. The holder was then placed on a precision rotating stage inside the chamber of an X-ray computed microtomography system (VersaXRM-500 High-Resolution 3D X-ray Tomography Microscope System, ZEISS® Xradia, Pleasanton, CA, USA). The distance between the samples and the detector was fixed at $20 \mathrm{~mm}$. In the measurements, the X-ray tube power and voltage were $3 \mathrm{~W}$ and $40 \mathrm{kV}$, respectively, with an exposure time of $20 \mathrm{~s}$. X-ray images with a field of view (FOV) of about $576 \mu \mathrm{m} \times 576 \mu \mathrm{m}$ were obtained with a $20 \times$ objective lens.

\section{Result and Discussion}

\subsection{Mechanical behaviour of core-shell microparticles}

Our next objective is investigating the mechanical strength of core-shell particles with liquid core under compression. Figure 5 compares the force-displacement relationships of the model and the experiment. The models can predict the deformation behaviour of the particle during compression as well as the maximum load a core-shell particle can bear. The force corresponding to a given displacement can be estimated using equation (2). Three sets of data from three core-shell geometries were prepared and investigated. As the core and the shell may not be centred (Fig. 6), the shell thickness is simply determined as the difference between the outer radius and the inner radius $h=b$ - $a$, Fig. 2(b). The ratios of the shell thickness to outer radius $h / b$ were $0.41,0.33$, and 0.21 , Figure 6 . 


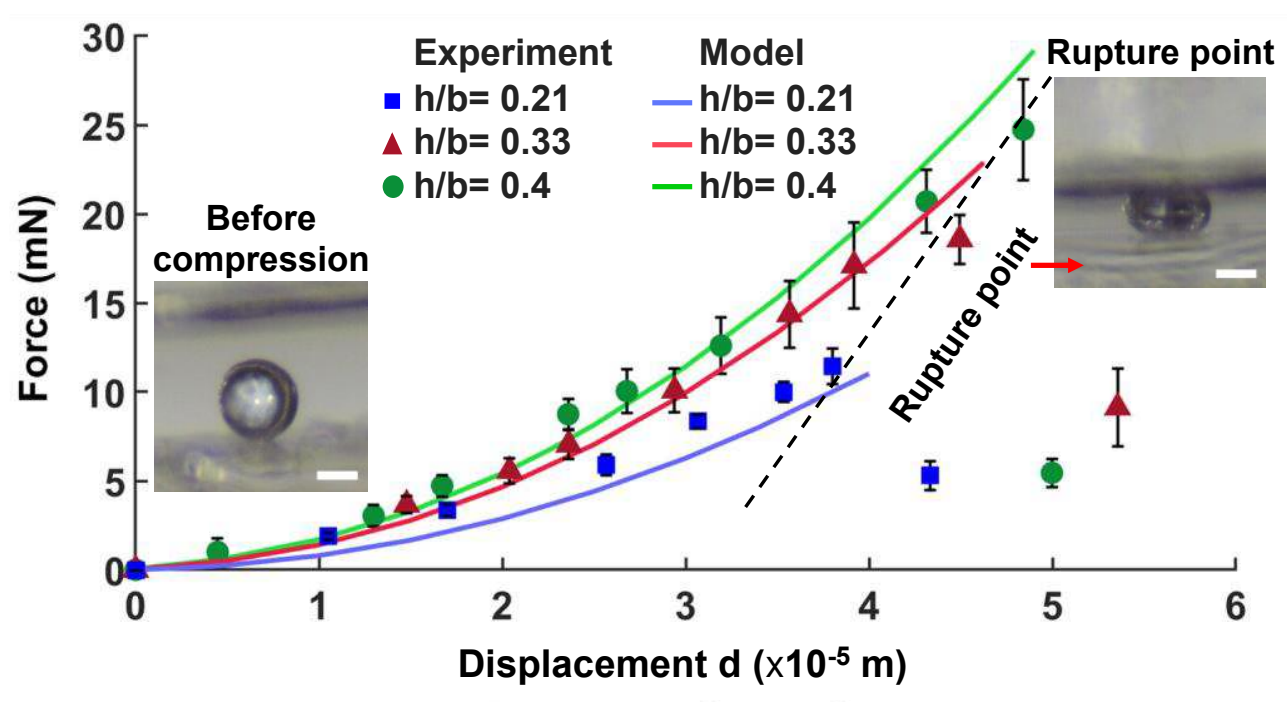

Figure 5. Force versus displacement characteristics of core-shell particles with different shell thickness to outer radius ratios $h / b$ of $0.41,0.33$, and 0.21 .

Figure 5 shows the force-displacement characteristics of these three particle geometries till rupture. The data were fitted into equation (2) with $A=0.022$ and $B=0.034$ and show an excellent agreement across all three geometries. The slight deviation of experimental data from the theoretical curve might be caused by asymmetry of the core liquid in the shell, as observed with X-ray images, Figure $6(\mathrm{~b})$. The average asymmetry is about $25 \%$ in the $\mathrm{x}-\mathrm{y}$ plane. Once the top plate touched the particle and compressed it, the force started to increase until reaching the critical displacement of rupture. The liquid contained in the shell causes it to bulge outwards and prevent the shell to buckle at the critical displacement. Subsequently, at a displacement beyond the critical rupture value, the liquid escapes and the force sharply dropped. Eventually, as the shell gets entirely compressed and the plate hits the weighing pan surface, the force again began to sharply increase. The critical displacement of rupture $x_{\max }$ was estimated from equation (3) by solving $U_{1}=U_{2}$ with $C=-0.001$. The corresponding rupture force of the critical displacement $d_{\max }$ can then be derived from equation (2). Figure 7(a) depicted the theoretical and experimental rupture force for $h / b$ ratios of $0.41,0.33$, and 0.21 . The rupture force increases with increasing ratio $h / b$. The results indicate that the rupture forces of the core-shell particles with $h / b$ ratios of 0.33 and 0.41 are almost 1.63 and 2.16 times greater than of the thins shell 
with $h / b=0.21$. Figure 7(b) shows the relationship between the critical displacement $d_{\max }$ and the ratio $h / b$. The critical rupture displacement increases with increasing ratio $h / b$.
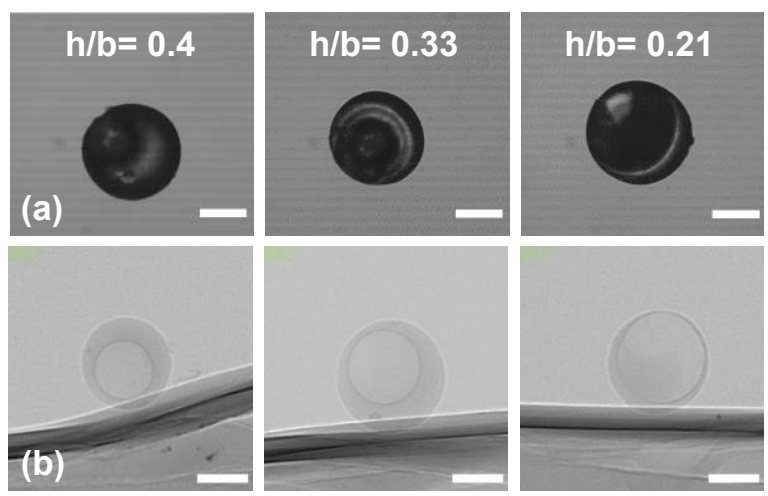

Figure 6. Images of core-shell microparticles used in the experiments: (a) Light microscopy; (b) X-ray computed microtomography. The shell thickness to outer radius ratios are $0.4,0.33$, and 0.21 . Scale bars represent $100 \mu \mathrm{m}$.
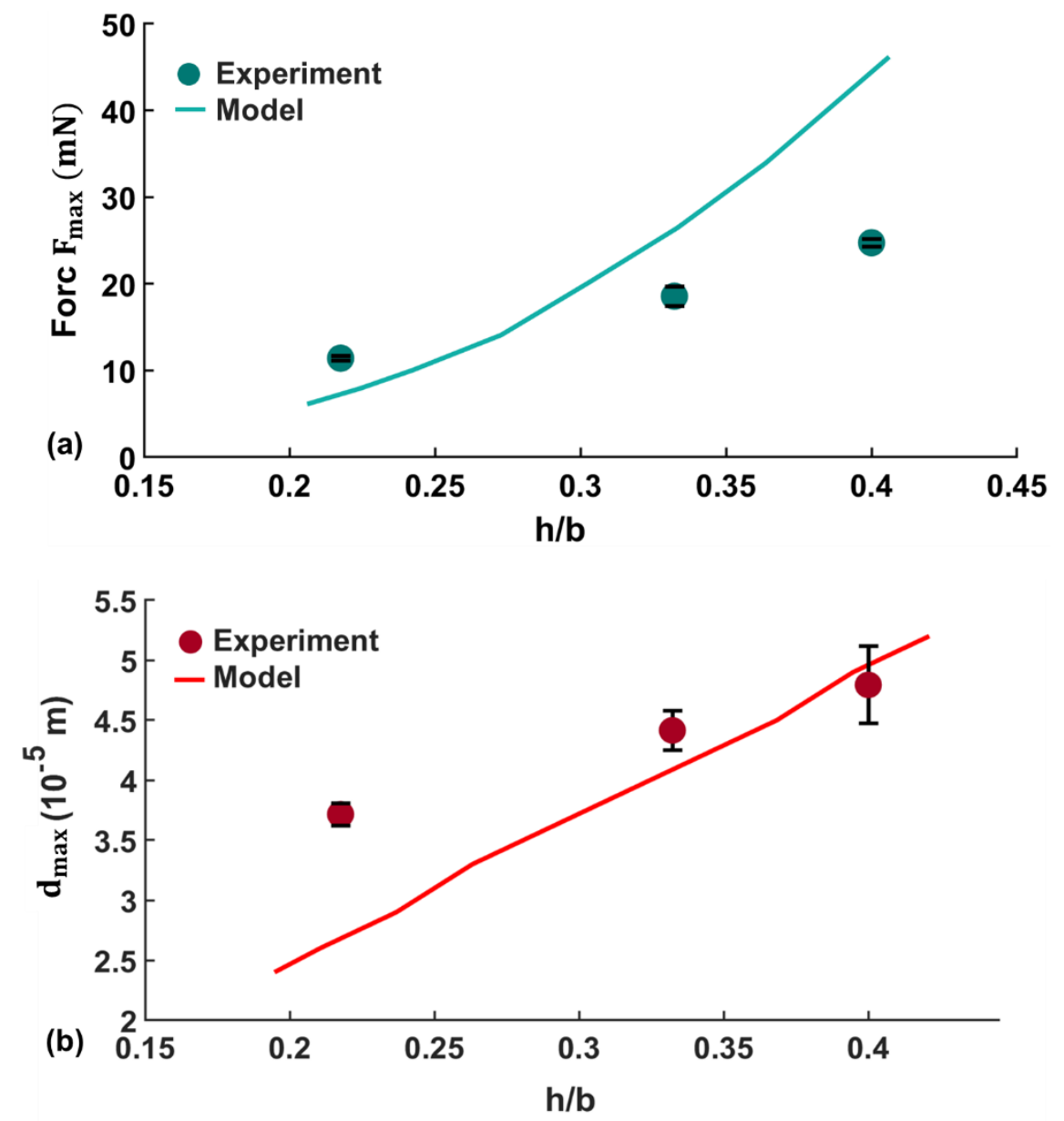
Figure 7. Critical rupture point as function of $h / b$ ratio: (a) Rupture force; (b) The critical displacement $\mathrm{d}_{\text {max }}$.

\subsection{Thermal behaviour of core-shell microparticles}

We first investigated the thermal behaviour of the core-shell microparticles experimentally by increasing the temperature and monitoring their response. Figure 8 shows representative core-shell microparticles with an outer diameter of approximately $196 \mu \mathrm{m}$ and a shell thickness of approximately $40 \mu \mathrm{m}$. The ratio between the thickness and the outer diameter is therefore approximately $h / b=0.41$. Figure 8 indicates no significant changes in the particles until the temperature reached $115^{\circ} \mathrm{C}$. The results imply that the core-shell particles maintain their shape and are stable up to this critical temperature. The polymeric shell encapsulating the oil core remained intact, without any leakage before reaching this temperature. According to Figure 8, the core-shell particles began to fracture and shrink after $115^{\circ} \mathrm{C}$. The gradual perforation of solid shell could be due to the expansion of the oil core encapsulated inside the particle upon heating. The expansion of the oil core causes the internal pressure to rise until it exceeds the tensile strength of the shell layer. ${ }^{[17]}$ After prolonged heating at temperature higher than the critical value, the core-shell particles appear to be hollow. Continuing to elevate the temperature causes the shell to turn brown and shrink. Figure 8 indicates that the core-shell particles were visibly destroyed at the temperature of $285^{\circ} \mathrm{C}$. We also repeated this experiment for core-shell particles with ratios of $h / b=0.21$ and $h / b=0.33$ to determine their critical rupture temperature. We utilised the rupture force values obtained from the compression test to estimate the critical temperatures of the core-shell particles during the heating process. The corresponding critical rupture temperatures could be obtained from Equation (9). Using the measured critical forces of Fig. 8(b), the critical rupture temperatures estimated with (10) for particles with $h / b$ ratios of $0.21,0.33$, and 0.41 are $62^{\circ} \mathrm{C}, 86^{\circ} \mathrm{C}$, and $109^{\circ} \mathrm{C}$, respectively. The experimental critical temperatures of the same set of $h / b$ ratios are $75 \pm 5^{\circ} \mathrm{C}, 95 \pm 5^{\circ} \mathrm{C}$ and 

critical temperature increases with increasing $h / b$ ratios or increasing shell thickness.

(a)

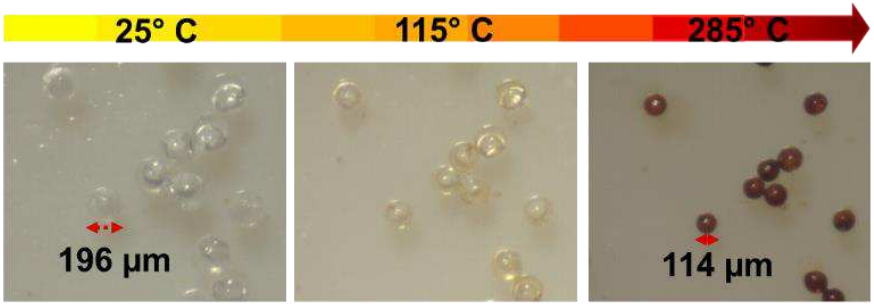

(b)

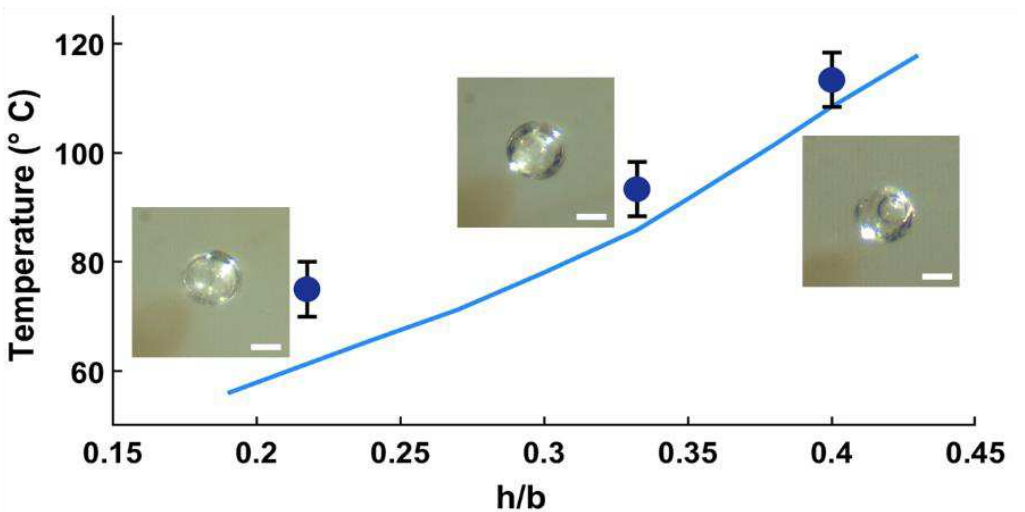

Figure 8. (a) Core-shell particles subjected to heating at elevated temperatures ranging from $25^{\circ} \mathrm{C}$ to $285^{\circ} \mathrm{C}$; (b) Critical rupture temperature as function of $h / b$ ratio. The above behaviour can be explained with the model of a thick-walled spherical shell filled with a liquid that induced a uniform pressure due to thermal expansion. The volume expansion coefficient $\alpha=1.29 \times 10^{-3} \mathrm{~K}^{-1}$ is assumed to remain constant. According to equation (9), there is a linear relationship between the pressure on inner wall of the shell and the temperature. The pressure difference to the ambient leads to a radial normal stress. The induced stress on the shell can be estimated using equation (11). For simplicity, the radial normal stress $\sigma_{\mathrm{r}}$ is considered zero on the outer surfaces. The maximum stress is at the inner surface at $r=a$, Fig. 2(b):

The critical maximum stress depends on the internal pressure proportional to the temperature of liquid core in the deformed state and the material properties of the core. The 
maximum stress our microparticles could endure is approximately $\sigma_{r, \max }=1.46 \mathrm{MPa}$ as estimated from equation (12).

\section{Conclusion}

We studied liquid core-shell particles subjected to thermal and compression loads. We established analytical models for the thermal and mechanical behaviour of thick-walled liquid core-shell particle under heating and compression. We found that the maximum internal pressure induced by the heated liquid core depends on the volume expansion coefficient and isothermal compressibility of the liquid. We note that a relatively thick shell made of TMPTMA and a thickness to ratio $h / b=0.41$ has a considerable thermal stability and can tolerate the thermal expansion of HFE7500 oil core up to $115^{\circ} \mathrm{C}$. In addition, we characterised the mechanical behaviour of this thick wall core-shell particles. The compression of the core-shell particle between two horizontal plates and the critical rupture force were modelled analytically. We conducted validation experiments with three different particle geometries. The forcedisplacement characteristics predicted by the analytical model agreed well with experimental data across all three particle geometries. Our findings suggest that the rupture behaviour of core-shell particles under compression depends on the ratio between the shell thickness and the outer radius. The rupture force increases with increasing thickness to radius ratio. In conclusion, the TMPTMA-based core-shell particles with a liquid core demonstrated good mechanical and thermal behaviour. These microparticles promise potential applications as capsule for storage and micro reaction.

\section{Conflicts of interest}

There are no conflicts of interest to declare.

\section{Acknowledgement}


The microfluidic device was fabricated in Queensland Microtechnology Facility, part of the Queensland node-Griffith of the Australian National Fabrication Facility, a company established under the National Collaborative Research Infrastructure Strategy to provide nano and microfabrication facilities for Australia's researchers. N. T. Nguyen acknowledges support from the Australian Research Council grant DP180100055.

\section{References}

[1] Galogahi, F.M., Y. Zhu, H.J. An, and N.T. Nguyen, Core-shell microparticles: Generation approaches and applications, Journal of Science-Advanced Materials and Devices. 5 (2020) 417-435. https://doi.org/10.1016/j.jsamd.2020.09.001.

[2] Yow, H.N. and A.F. Routh, Formation of liquid core-polymer shell microcapsules, Soft Matter. 2 (2006) 940-949. https://doi.org/10.1039/b606965g.

[3] Li, Q., X. Liu, X. Wang, S. Qiu, K. Byambasuren, L. Dang, and Z. Wang, Antiproliferative ability and fluorescence tracking of $\alpha$-linolenic acid-loaded microemulsion as label-free delivery carriers in MDA-MB-231 cells, Journal of agricultural and food chemistry. $67 \quad$ (2019) 11518-11526. https://doi.org/10.1021/acs.jafc.9b04972.

[4] Tiribocchi, A., A. Montessori, M. Lauricella, F. Bonaccorso, S. Succi, S. Aime, M. Milani, and D.A. Weitz, The vortex-driven dynamics of droplets within droplets, Nature Communications. 12 (2021) 1-10. https://doi.org/ARTN 82

10.1038/s41467-020-20364-0.

[5] Wang, H., Z. Zhao, Y. Liu, C. Shao, F. Bian, and Y. Zhao, Biomimetic enzyme cascade reaction system in microfluidic electrospray microcapsules, Sci Adv. 4 (2018) eaat2816. https://doi.org/10.1126/sciadv.aat2816. 
[6] Nguyen, N.T., S. Schubert, S. Richter, and W. Dotzel, Hybrid-assembled micro dosing system using silicon-based micropump/valve and mass flow sensor, Sensors and Actuators a-Physical. 69 (1998) 85-91. $\quad$ https://doi.org/Doi 10.1016/S09244247(98)00039-9.

[7] Dinh, T., H.P. Phan, A. Qamar, P. Woodfield, N.T. Nguyen, and D.V. Dao, Thermoresistive Effect for Advanced Thermal Sensors: Fundamentals, Design Considerations, and Applications, Journal of Microelectromechanical Systems. 26 (2017) 966-986. https://doi.org/10.1109/Jmems.2017.2710354.

[8] Dinh, T., H.P. Phan, D.V. Dao, P. Woodfield, A. Qamara, and N.T. Nguyen, Graphite on paper as material for sensitive thermoresistive sensors, Journal of Materials Chemistry C. 3 (2015) 8776-8779. https://doi.org/10.1039/c5tc01650a.

[9] Bandara, T., N.T. Nguyen, and G. Rosengarten, Slug flow heat transfer without phase change in microchannels: A review, Chemical Engineering Science. 126 (2015) 283295. https://doi.org/10.1016/j.ces.2014.12.007.

[10] Mytnyk, S., I. Ziemecka, A.G.L. Olive, J.W.M. van der Meer, K.A. Totlani, S. Oldenhof, M.T. Kreutzer, V. van Steijn, and J.H. van Esch, Microcapsules with a permeable hydrogel shell and an aqueous core continuously produced in a 3D microdevice by all-aqueous microfluidics, Rsc Advances. 7 (2017) 11331-11337. https://doi.org/10.1039/c7ra00452d.

[11] Shchukin, D.G., G.B. Sukhorukov, and H. Möhwald, Smart inorganic/organic nanocomposite hollow microcapsules, Angewandte Chemie International Edition. 42 (2003) 4472-4475. https://doi.org/10.1002/anie.200352068.

[12] Mercadé-Prieto, R., R. Allen, Z. Zhang, D. York, J.A. Preece, and T.E. Goodwin, Failure of elastic-plastic core-shell microcapsules under compression, AIChE journal. 58 (2012) 2674-2681. https://doi.org/10.1002/aic.12804. 
[13] Fujiwara, M., K. Shiokawa, K. Morigaki, Y.C. Zhu, and Y. Nakahara, Calcium carbonate microcapsules encapsulating biomacromolecules, Chemical Engineering Journal. 137 (2008) 14-22. https://doi.org/10.1016/j.cej.2007.09.010.

[14] Caruso, M.M., B.J. Blaiszik, H. Jin, S.R. Schelkopf, D.S. Stradley, N.R. Sottos, S.R. White, and J.S. Moore, Robust, double-walled microcapsules for self-healing polymeric materials, ACS Appl Mater Interfaces. 2 (2010) 1195-9. https://doi.org/10.1021/am100084k.

[15] Kang, S., M. Baginska, S.R. White, and N.R. Sottos, Core-shell polymeric microcapsules with superior thermal and solvent stability, ACS Appl Mater Interfaces. 7 (2015) 10952-6. https://doi.org/10.1021/acsami.5b02169.

[16] Fung, W.T., A. Beyzavi, P. Abgrall, N.T. Nguyen, and H.Y. Li, Microfluidic platform for controlling the differentiation of embryoid bodies, Lab Chip. 9 (2009) 2591-5. https://doi.org/10.1039/b903753e.

[17] Vasudevan, D., R.R. Gaddam, A. Trinchi, and I. Cole, Core-shell quantum dots: Properties and applications, Journal of Alloys and Compounds. 636 (2015) 395-404. https://doi.org/10.1016/j.jallcom.2015.02.102.

[18] Liu, S., S.N. Reed, M.J. Higgins, M.S. Titus, and R. Kramer-Bottiglio, Oxide ruptureinduced conductivity in liquid metal nanoparticles by laser and thermal sintering, Nanoscale. 11 (2019) 17615-17629. https://doi.org/10.1039/c9nr03903a.

[19] Latnikova, A. and A. Yildirim, Thermally induced release from polymeric microparticles with liquid core: the mechanism, Soft matter. 11 (2015) 2008-2017. https://doi.org/10.1039/C4SM02674H.

[20] Zhao, H., X. Fei, L. Cao, S. Zhao, and J. Zhou, Changes in microcapsules under heating: the effect of particle size on thermal stability and breakability, Journal of Materials Science. 55 (2020) 3902-3911. https://doi.org/10.1007/s10853-019-04297-8. 
449 [21] Leopércio, B.C., M. Michelon, and M.S. Carvalho, Deformation and rupture of

450

451

452

453

454

455

456

457

458

459

460

461

462

463

464

465

466

467

468

469

470

471

472 microcapsules flowing through constricted capillary, Scientific Reports. 11 (2021) 112. https://doi.org/10.1038/s41598-021-86833-8.

[22] Perez, R.A. and H.W. Kim, Core-shell designed scaffolds for drug delivery and tissue engineering,

Acta

Biomater.

21

(2015)

2-19. https://doi.org/10.1016/j.actbio.2015.03.013.

[23] Neubauer, M.P., M. Poehlmann, and A. Fery, Microcapsule mechanics: from stability to function, Adv Colloid Interface Sci. $207 \quad$ (2014) 65-80. https://doi.org/10.1016/j.cis.2013.11.016.

[24] Zhang, G.S., Z, Mechanical properties of melamine-formaldehyde microcapsules, Journal of microencapsulation. 18 (2001) 593-602. https://doi.org/ $10.1080 / 02652040010019541$.

[25] Sun, G. and Z. Zhang, Mechanical strength of microcapsules made of different wall materials, International Journal of Pharmaceutics. 242 (2002) 307-311. https://doi.org/Pii S0378-5173(02)00193-X

Doi 10.1016/S0378-5173(02)00193-X.

[26] Risso, F. and M. Carin, Compression of a capsule: mechanical laws of membranes with negligible bending stiffness, Phys Rev E Stat Nonlin Soft Matter Phys. 69 (2004) 061601. https://doi.org/10.1103/PhysRevE.69.061601.

[27] Liu, K.K., D.R. Williams, and B.J. Briscoe, Compressive deformation of a single microcapsule, Phys Rev E Stat Phys Plasmas Fluids Relat Interdiscip Topics. 54 (1996) 6673-6680. https://doi.org/10.1103/physreve.54.6673.

[28] Carin, M., D. Barthes-Biesel, F. Edwards-Levy, C. Postel, and D.C. Andrei, Compression of biocompatible liquid-filled HSA-alginate capsules: determination of 
the membrane mechanical properties, Biotechnol Bioeng. 82 (2003) 207-12. https://doi.org/10.1002/bit.10559.

[29] Ansari, A. and M. Abbaspour, Modelling and economic evaluation of pressure-retarded osmosis power plant case study: Iran, International Journal of Ambient Energy. 40 (2019) 69-81. https://doi.org/10.1080/01430750.2017.1354323.

[30] Bando, K., K. Ohba, and Y. Oiso, Deformation analysis of microcapsules compressed by two rigid parallel plates, Journal of biorheology. 27 (2013) 18-25. https://doi.org/10.1007/s12573-012-0053-8.

[31] Taber, L.A., Large Deflection of a Fluid-Filled Spherical-Shell under a Point Load, Journal of Applied Mechanics-Transactions of the Asme. 49 (1982) 121-128. https://doi.org/Doi 10.1115/1.3161953.

[32] Rachik, M., D. Barthes-Biesel, M. Carin, and F. Edwards-Levy, Identification of the elastic properties of an artificial capsule membrane with the compression test: effect of thickness, J Colloid Interface Sci. $301 \quad$ (2006) 217-26. https://doi.org/10.1016/j.jcis.2006.04.062.

[33] Teo, A.J.T., F. Malekpour-Galogahi, K.R. Sreejith, T. Takei, and N.T. Nguyen, Surfactant-free, UV-curable core-shell microcapsules in a hydrophilic PDMS $\begin{array}{llllll}\text { microfluidic } & \text { device, } & \text { Aip } & \text { Advances. } & 10 & \text { (2020) }\end{array}$ https://doi.org/10.1063/5.0004736.

[34] Galogahi, F.M., Y. Zhu, H. An, and N.-T. Nguyen, Formation of core-shell droplets for the encapsulation of liquid contents, Microfluidics and Nanofluidics. 25 (2021) 1-11. https://doi.org/10.1007/s10404-021-02483-2.

[35] Pauchard, L. and S. Rica, Contact and compression of elastic spherical shells: the physics of a 'ping-pong'ball, Philosophical Magazine B. 78 (1998) 225-233. https://doi.org/10.1080/13642819808202945. 
[36] Shorter, R., J. Smith, V. Coveney, and J. Busfield, Axial compression of hollow elastic spheres, Journal of Mechanics of Materials and Structures. 5 (2010) 693-705. https://doi.org/10.2140/jomms.2010.5.693.

[37] Young, W.C., R.G. Budynas, and A.M. Sadegh, Roark's formulas for stress and strain, ed., McGraw-Hill Education, 2012.

[38] Borgnakke, C. and R.E. Sonntag, Fundamentals of thermodynamics, ed., John Wiley \& Sons, 2020.

[39] Fang, D., Y. Li, X. Meng, and J. Wu, Liquid density of HFE-7200 and HFE-7500 from $\mathrm{T}=(283$ to 363$) \mathrm{K}$ at pressures up to $100 \mathrm{MPa}$, The Journal of Chemical Thermodynamics. 69 (2014) 36-42. https://doi.org/10.1016/j.jct.2013.09.035.

[40] Sreejith, K.R., L. Gorgannezhad, J. Jin, C.H. Ooi, T. Takei, G. Hayase, H. Stratton, K. Lamb, M. Shiddiky, D.V. Dao, and N.T. Nguyen, Core-Shell Beads Made by Composite Liquid Marble Technology as A Versatile Microreactor for Polymerase Chain Reaction, Micromachines (Basel). 11 (2020) 242. https://doi.org/10.3390/mi11030242. 


\section{Supplementary Files}

This is a list of supplementary files associated with this preprint. Click to download.

- SupportingInformation1.docx 